\title{
Electronic Content
}

National Cancer Institute

\section{Source}

National Cancer Institute. Electronic Content. NCI Thesaurus. Code C99094.

Material recorded in a format that requires an electronic device to view or modify, encompassing documents, images, multimedia, web pages, and other digital media. 